\title{
A one-dimensional model exhibiting phase transition
}

\author{
A.A. Kerimov*, M.A. Şahin \\ Department of Mathematics, Bilkent University, 06533 Bilkent, Ankara, Turkey
}

Received 12 May 1998; received in revised form 19 October 1998

\begin{abstract}
A one-dimensional model with two spin variables having a unique ground state and at least two extreme limit Gibbs states is constructed. (c) 1999 Elsevier Science B.V. All rights reserved.
\end{abstract}

\section{Introduction}

The problem of phase transitions in one-dimensional models has been studied in various papers [1-16]. In the present paper, we construct a one-dimensional model with a unique ground state having at least two extreme Gibbs states at $\beta=1$ which makes clear two questions formulated before. In [14] the following conjecture was formulated: any one-dimensional model with discrete spin space and with a unique ground state has a unique Gibbs state if either the spin space of this model is finite or the potential of this model is translationally invariant. The arguments for this conjecture which originates from [13] are listed in [15]. Examples of models exhibiting phase transition in cases when the conditions of the conjecture are violated are constructed in $[14,15]$. In these papers, one-dimensional models with a unique ground state, and non translation invariant potential and countable spin space having, respectively, at least two and countable many extreme Gibbs states are constructed. Both models have the following property. If $\mathbf{P}^{\alpha}$ is an extreme limit Gibbs state "corresponding" to the spin $\alpha($ see $[15])$ then

$$
\mathbf{P}^{\alpha}\left(\varphi\left(x_{1}\right)=\alpha, \varphi\left(x_{2}\right)=\alpha, \ldots, \varphi\left(x_{l}\right)=\alpha\right)>1 / 2,
$$

where the last inequality is held uniformly with respect to $l$ and $x_{1}, x_{2}, \ldots, x_{l}$.

The last inequality is unusual for random fields but on the other hand is typical for the one-dimensional models with short-range interaction exhibiting phase transition: in

* Corresponding author. E-mail: kerimov@fen.bilkent.edu.tr. 
[9] it is shown that the inhomogeneous Ising models exhibiting phase transition have the property (1). The explanation of property (1) is that in one-dimensional short-range models exhibiting a phase transition the coupling potential is strong enough to guarantee the inequality (1).

The natural question arises [15]: is the property (1) held necessarily for any onedimensional model with unique ground state exhibiting phase transition?

In this paper, we construct a model having two spins and a unique ground state exhibiting a phase transition for which the property (1) does not hold. Thus,

(a) the answer for the last question is negative,

(b) the Conjecture as formulated in [14] is not true.

We say that the ground state $\varphi^{g r}(x)$ is "stable", if for any finite set $A \subset \mathbf{Z}^{1}$ with the length $|A|$

$$
H\left(\varphi^{\prime}(x)\right)-H\left(\varphi^{g r}(x)\right) \geqslant t|A|,
$$

where $t>0,|A|$ is the number of sites of $A$ and $\varphi^{\prime}(x)$ is a perturbation of the ground state $\varphi^{g r}$ on the finite set $A$.

In spite of the counterexample constructed in the present paper, the conjecture is valid under some natural additional conditions:

Let $H(\varphi(x))=\sum_{B \subset \mathbf{Z}^{1}} U(\varphi(B))$. The value of the interaction of the contour $K_{0}$ with the contours $K_{1}, \ldots, K_{n}$ we denote via $G\left(K_{0} \mid K_{1}, \ldots, K_{n}\right)$ :

$$
G\left(K_{0} \mid K_{1}, \ldots, K_{n}\right)=\prod_{B \in I G(0 \mid 1, \ldots, n)}(1+\exp (-\beta f(B)-1)),
$$

where $I G(0 \mid 1, \ldots, n)$ is the set of all interaction elements intersecting the support of the contour $K_{0}$.

On the potential $U(B)$ we impose the following natural condition:

$$
\begin{aligned}
G\left(K_{0} \mid K_{1}, \ldots, K_{n}\right)= & \prod_{\substack{B \in I G(0 \mid 1, \ldots, n)\\
}} \mid(1+\exp (-\beta f(B)-1)) \\
& \leqslant h_{1}\left(\left|\operatorname{supp}\left(K_{0}\right)\right|\right) h_{2}(\operatorname{dist}(0 \mid 1, \ldots, n)),
\end{aligned}
$$

where $\operatorname{dist}(0 \mid 1, \ldots, n)$ is the distance between the support of $K_{0}$ and the union of the supports of contours $K_{1}, \ldots, K_{n}$, and the functions $h_{i}(x)$ satisfy the following conditions:

$$
\lim _{x \rightarrow \infty} h_{1}(x) / x=0, \quad \lim _{x \rightarrow \infty} h_{2}(x)=0 .
$$

In other words, the interaction of $K_{1}, \ldots, K_{n}$ on $K_{0}$ tends to zero when the distance between them increases, and the value of the interaction increases with a rate less than the length of the support of $K_{0}$.

These conditions are very natural and in particular are held in all models with pair potential $U(x) \sim 1 / x^{1+\alpha}$, as $x \rightarrow \infty, 0<\alpha$. In the pair potential case (see [13])

$$
G\left(K_{0} \mid K_{1}, \ldots, K_{n}\right) \leqslant \operatorname{const}(\operatorname{dist}(0 \mid 1, \ldots, n))^{-\alpha}\left(\left|\operatorname{supp}\left(K_{0}\right)\right|\right)^{1-\alpha} .
$$

Theorem 1. Consider a one-dimensional model with finite spin space and with a unique "stable" ground state. Suppose that the potential satisfies above formulated 
decreasing conditions. Then the model has a unique Gibbs state at low temperatures [16].

\section{A model}

In this section, we construct a model with a unique ground state and two spin variables which has at least two extreme limit Gibbs states.

Consider a partition of $\mathbf{Z}^{1} \cap(-\infty,-1]$ into intervals $I_{n}=\left[a_{n+1}, a_{n}\right], n=1,2, \ldots$, where the sequence $a_{n}$ is defined by the following recurrence relation:

$$
a_{1}=-0.5 \quad a_{n}-a_{n+1}=b_{n}=\left(1-0.99^{0.5^{n-1}}\right)^{-1} .
$$

The model is defined by the Hamiltonian

$$
H(\varphi(x))=\sum_{x \in Z^{1} ; x<0} U\left(\varphi(x), \varphi\left(I_{n+1}\right)\right)-\sum_{x \in Z^{1} ; x \geqslant 0} \varphi(x),
$$

where the value of $n$ is defined by the condition $x \in I_{n}$ and the spin variables $\varphi(x)$ take two values: 1 and 0 .

The potential $U$ is defined by the following formulas:

$$
\begin{aligned}
& U\left(\varphi(x)=1, \varphi\left(I_{n+1}\right)\right)=-\ln 0.8 \text { if } \sum_{x \in Z^{1} ; x \in I_{n+1}} \varphi(x) / b_{n+1} \geqslant 0.7, \\
& U\left(\varphi(x)=0, \varphi\left(I_{n+1}\right)\right)=-\ln 0.2 \text { if } \sum_{x \in Z^{1} ; x \in I_{n+1}} \varphi(x) / b_{n+1} \geqslant 0.7, \\
& U\left(\varphi(x)=1, \varphi\left(I_{n+1}\right)\right)=-\ln 0.6 \text { if } \sum_{x \in Z^{1} ; x \in I_{n+1}} \varphi(x) / b_{n+1}<0.7, \\
& U\left(\varphi(x)=0, \varphi\left(I_{n+1}\right)\right)=-\ln 0.4 \quad \text { if } \sum_{x \in Z^{1} ; x \in I_{n+1}} \varphi(x) / b_{n+1}<0.7 .
\end{aligned}
$$

Lemma 1. The configuration $\varphi^{g r}(x)=1$ is the unique ground state of the model (4).

Proof. Let a configuration $\varphi^{\prime}(x)$ be a finite perturbation of the configuration $\varphi(x)=1$. Then

$$
\begin{aligned}
& H\left(\varphi^{\prime}(x)\right)-H(\varphi(x)) \\
& \quad=\sum_{x \in Z^{1} ; x \leqslant 0}\left(U\left(\varphi^{\prime}(x), \varphi^{\prime}\left(I_{n+1}\right)\right)-U\left(\varphi(x), \varphi\left(I_{n+1}\right)\right)\right)-\sum_{x \in Z^{1} ; x>0}\left(\varphi^{\prime}(x)-\varphi(x)\right) \\
& \quad=\sum_{1}-\sum_{2}>0
\end{aligned}
$$


since due to the definitions possible nonzero terms of $\sum_{1}$ are $-\ln 0.8-\ln 0.8,-\ln 0.6-$ $\ln 0.8,-\ln 0.4-\ln 0.8$ and $-\ln 0.2-\ln 0.8$ and they are nonnegative and all nonzero terms of $\sum_{2}$ are $1-1$ and $0-1$ and they are nonpositive. Therefore, the configuration $\varphi^{g r}(x)=1$ is a ground state.

Now, let the configuration $\varphi^{\prime}(x)$ be a ground state. We show that for any $x^{\prime} \in$ $Z^{1} \varphi^{\prime}\left(x^{\prime}\right)=1$. Indeed, if $x^{\prime} \geqslant 0$ and $\varphi^{\prime}\left(x^{\prime}\right)=0$, we define a configuration $\varphi^{\prime \prime}(x)$ by the formula: $\varphi^{\prime \prime}\left(x^{\prime}\right)=1$ and for all $x \neq x^{\prime} \varphi^{\prime \prime}(x)=\varphi^{\prime}(x)$. Then $H\left(\varphi^{\prime \prime}(x)\right)-H\left(\varphi^{\prime}(x)\right)=-1<0$ and contradiction. On the other hand, if $x^{\prime}<0$ and $\varphi^{\prime}\left(x^{\prime}\right)=0$, we define a configuration $\varphi^{\prime \prime}(x)$ by the formula: $\varphi^{\prime \prime}(x)=1$ for all $x^{\prime} \leqslant x \leqslant 0$ and $\varphi^{\prime \prime}(x)=\varphi^{\prime}(x)$ for all $x \notin\left[x^{\prime}, 0\right]$. Then, as can be easily shown $H\left(\varphi^{\prime \prime}(x)\right)-H\left(\varphi^{\prime}(x)\right)<0$ and again contradiction. The lemma is proved.

Theorem 2. Let $\beta=1$. There exist at least two limit Gibbs states of the model (1).

Proof. Consider limit Gibbs states $\mathbf{P}^{0}$ and $\mathbf{P}^{1}$ corresponding to the boundary conditions $\varphi^{0}(x)$ and $\varphi^{1}(x)$, respectively, where $\varphi^{0}(x)=0$ and $\varphi^{1}(x)=1$. In order to prove the theorem we show that

$$
\begin{aligned}
& \mathbf{P}^{0}(\varphi(-1)=0)>0.59, \\
& \mathbf{P}^{1}(\varphi(-1)=1)>0.79 .
\end{aligned}
$$

We start with the proof of inequality (6).

Since $\mathbf{P}^{1}$ and $\mathbf{P}^{0}$ are weak limits of $\mathbf{P}_{V}^{1}$ and $\mathbf{P}_{V}^{0}$ in order to prove the inequalities (5) and (6) we show that

$$
\begin{aligned}
& \mathbf{P}_{V}^{0}(\varphi(-1)=1)>0.594, \\
& \mathbf{P}_{V}^{1}(\varphi(-1)=1)>0.792,
\end{aligned}
$$

where $\mathbf{P}_{V}^{1}$ and $\mathbf{P}_{V}^{0}$ are the Gibbs distributions corresponding, respectively, to the boundary conditions $\varphi^{1}(x)=1$ and $\varphi^{0}(x)=0, x \in \mathbf{Z}^{1}-[-V, V]$.

We start with the proof of inequality (8).

Let a configuration $\varphi(x)$ be fixed. We say that the interval $I_{n}$ is 1-good, if $\sum_{x \in Z^{1} ; x \in I_{n}}$ $\times \varphi(x) / b_{n} \geqslant 0.7$.

It follows from the definition of the Hamiltonian that since all spin variables $\varphi(x), x \in$ $[0, V]$ are independent and do not depend on the boundary conditions, the restriction of the Gibbs distribution $\mathbf{P}_{V}^{1}$ to the set $\varphi(x), x \in[-V,-1]$ can be treated as a Markov chain starting at point $x=-V$ and ending at point $x=-1$ with the following transition probabilities (the memory of the Markov chain tends to infinity when $V$ tends to infinity):

$$
\begin{aligned}
& \mathbf{P}_{V}^{1}\left(\varphi(x)=1 \mid I_{n+1} \text { is } 1 \text {-good }\right)=0.8, \\
& \mathbf{P}_{V}^{1}\left(\varphi(x)=1 \mid I_{n+1} \text { is not 1-good }\right)=0.6 .
\end{aligned}
$$


First of all, note that

$$
\begin{aligned}
\mathbf{P}_{V}^{1}(\varphi(-1)=1)= & \mathbf{P}_{V}^{1}\left(\varphi(-1)=1 \cap I_{2} \text { is } 1 \text {-good }\right) \\
& +\mathbf{P}_{V}^{1}\left(\varphi(-1)=1 \cap I_{2} \text { is not } 1 \text {-good }\right) \\
\geqslant & \mathbf{P}_{V}^{1}\left(\varphi(-1)=1 \cap I_{2} \text { is } 1 \text {-good }\right)=\mathbf{P}_{V}^{1}(\varphi(-1) \\
= & \left.1 \mid I_{2} \text { is } 1 \text {-good }\right) \mathbf{P}_{V}^{1}\left(I_{2} \text { is } 1 \text {-good }\right) \\
= & 0.8 \mathbf{P}_{V}^{1}\left(I_{2} \text { is } 1 \text {-good }\right) .
\end{aligned}
$$

Thus, in order to prove (8), it is sufficient to show that

$$
\mathbf{P}_{V}^{1}\left(I_{2} \text { is } 1 \text {-good }\right)>0.99 \text {. }
$$

Suppose that $[-V-1 / 2,-1 / 2]=\bigcup_{k=1}^{l} I_{k}$.

Since $\mathbf{P}_{V}^{1}\left(I_{2}\right.$ is 1 -good $) \geqslant \mathbf{P}_{V}^{1}\left(\bigcap_{k=2}^{l}\left(I_{k}\right.\right.$ is 1 -good $\left.)\right)$, in order to prove (9) we prove that

$$
\mathbf{P}_{V}^{1}\left(\bigcap_{k=2}^{l}\left(I_{k} \text { is } 1 \text {-good }\right)\right)>0.99 .
$$

Now, note that

$$
\begin{aligned}
\mathbf{P}_{V}^{1} & \left(\bigcap_{k=2}^{l}\left(I_{k} \text { is } 1 \text {-good }\right)\right) \\
= & \mathbf{P}_{V}^{1}\left(I_{l} \text { is } 1 \text {-good } \mid \varphi(x)=1, x<-V\right) \\
& \left.\times \prod_{k=l-1}^{2} \mathbf{P}_{V}^{1}\left(I_{k} \text { is } 1 \text {-good }\right) \mid I_{k+1} \text { is } 1 \text {-good }\right) .
\end{aligned}
$$

We estimate the probability $\mathbf{P}_{V}^{1}\left(I_{k}\right.$ is $\left.1-\operatorname{good}\right) \mid I_{k+1}$ is $\left.1-\operatorname{good}\right)$.

$$
\begin{aligned}
\left.\mathbf{P}_{V}^{1}\left(I_{k} \text { is } 1 \text {-good }\right) \mid I_{k+1} \text { is } 1 \text {-good }\right) \\
=\mathbf{P}_{V}^{1}\left(\sum_{x \in Z^{1} ; x \in I_{k}} \varphi(x) / b_{k} \geqslant 0.7 \mid I_{k+1} \text { is } 1 \text {-good }\right) \\
\geqslant \mathbf{P}_{V}^{1}\left(\left|\sum_{x \in Z^{1} ; x \in I_{k}} \varphi(x) / b_{k}-0.8\right|<0.5\right) .
\end{aligned}
$$

Now, note that the Markov chain $\mathbf{P}_{V}^{1}\left(\varphi\left(I_{k}\right)\right) \mid I_{k+1}$ is1-good) starting at point $a_{n+1}+1 / 2$ and ending at point $a_{n}-1 / 2$ can be treated as a sequence of independent Bernoulli random variables taking the values 1 and 0 with probabilities 0.8 and 0.2 . Thus, by applying the Weak Law of Large Numbers to the last expression we get

$$
\begin{gathered}
\left.\mathbf{P}_{V}^{1}\left(\left|\sum_{x \in Z^{1} ; x \in I_{k}} \varphi(x) / b_{k}-0.8\right|<0.5\right\rangle\right)>1-\frac{0.80 .2}{0.25 b_{k}} \\
>1-1 / b_{k}=1-\left(1-0.99^{0.5^{k-1}}\right)=0.99^{0.5^{k-1}} .
\end{gathered}
$$


Now

$$
\begin{aligned}
\mathbf{P}_{V}^{1} & \left(\bigcap_{k=2}^{l}\left(I_{k} \text { is } 1 \text {-good }\right)\right) \\
= & \mathbf{P}_{V}^{1}\left(I_{l} \text { is } 1-\operatorname{good} \mid \varphi(x)=1, x<-V\right) \\
& \times \prod_{k=l-1}^{2} \mathbf{P}_{V}^{1}\left(I_{k} \text { is } 1-\operatorname{good} \mid I_{k+1} \text { is } 1 \text {-good }\right) \\
& \geqslant \prod_{k=2}^{\infty} \mathbf{P}_{V}^{1}\left(I_{k} \text { is } 1 \text {-good } \mid I_{k+1} \text { is } 1 \text {-good }\right) \geqslant \prod_{k=2}^{\infty} 0.99^{0.5^{k-1}}=0.99 .
\end{aligned}
$$

Thus, inequality (10) is proved. Now, inequality (10) implies inequality (8) which in its turn implies inequality (6).

Inequality (7) has a similar proof, we do not go into details. We say that the interval $I_{n}$ is 0 -good, if $\sum_{x \in Z^{1} ; x \in I_{n}} \varphi(x) / b_{n}<0.7$.

The only difference with the proof of (6) is that the Markov chain $\mathbf{P}_{V}^{0}\left(\varphi\left(I_{k}\right)\right) \mid I_{k+1}$ is 0 -good) is a sequence of independent Bernoulli random variables taking the values 1 and 0 with probabilities 0.6 and 0.4 . But this difference is not essential, since

$$
\begin{aligned}
\left.\mathbf{P}_{V}^{0}\left(I_{k} \text { is } 0 \text {-good }\right) \mid I_{k+1} \text { is } 0 \text {-good }\right) & =\mathbf{P}_{V}^{0}\left(\sum_{x \in Z^{1} ; x \in I_{k}} \varphi(x) / b_{k}<0.7 \mid I_{k+1} \text { is } 0 \text {-good }\right) \\
& \geqslant \mathbf{P}_{V}^{0}\left(\left|\sum_{x \in Z^{1} ; x \in I_{k}} \varphi(x) / b_{k}-0.6\right|<0.5\right) \\
& \geqslant \mathbf{P}_{V}^{0}\left(\left|\sum_{x \in Z^{1} ; x \in I_{k}} \varphi(x) / b_{k}-0.6\right|<0.5\right)>1-\frac{0.60 .4}{0.25 b_{k}} \\
& >1-1 / b_{k}=1-\left(1-0.99^{0.5^{k-1}}\right)=0.99^{0.5^{k-1}} .
\end{aligned}
$$

Theorem 2 is proved.

\section{Final remarks}

The model evidently does not satisfy (1).

The unique ground state of the model (4) is "stable"! Indeed, suppose that a configuration $\varphi^{\prime}(x)$ is a perturbation of the ground state $\varphi^{g r}(x)$ on some finite area $A$. If $A \subset[0, \infty)$, then it can be easily shown that inequality (2) is held with $t=1$. Suppose that the set $A \subset(-\infty, 0)$. Let us arrange the elements of $A$ into decreasing order: $A=x_{1}, x_{2}, \ldots, x_{p}$, where $x_{i}>x_{j}$ if $i<j$. Consider the expression $\Delta=H\left(\varphi^{\prime}(x)\right)-$ $H\left(\varphi^{g r}(x)\right)$. Suppose that $x_{1} \in I_{n}$. When we replace the $\varphi^{\prime}\left(x_{1}\right)=0$ by 1 , then by the definitions the $\Delta$ becomes less by either $-\ln 0.2+\ln 0.8$ (if the interval $I_{n+1}$ is 1 -good) 
or $-\ln 0.4+\ln 0.6$ (if the interval $I_{n+1}$ is 0 -good). After that we replace the $\varphi\left(x_{2}\right)=0$ by 1 , again the $\Delta$ becomes less by the same rule. Also note that, if the configuration $\varphi^{\prime}(x)$ contains some number of 0-good intervals, then after some number of steps each 0-good interval becomes 1-good interval, and in this transition, when the interval $I_{n}$ becomes 1 -good the $\Delta$ again becomes less by $-\ln 0.6+\ln 0.8$ times the length of $I_{n-1}$.

After $p$ steps the value of $\Delta$ evidently becomes 0 . Therefore,

$$
H\left(\varphi^{\prime}(x)\right)-H\left(\varphi^{g r}(x)\right) \geqslant t|A|,
$$

where $t=\min (1,-\ln 0.2+\ln 0.8,-\ln 0.4+\ln 0.6)=\ln 1.5$.

Thus, the ground state $\varphi^{g r}(x)$ is stable, but there is a phase transition. The reason of this fact is the following: if we define contours in the model (4) as connected collections of spin variables $\varphi(x)=0$, then one can easily verify that the "interaction" of fixed contour $K$ with the boundary and other contours does not satisfy the conditions of Theorem 1 .

Recently one of the authors (A.K.) proved Theorem 1 at all temperatures. Thus, in one-dimensional models "stability" of ground state is important at all temperatures.

\section{Acknowledgements}

The authors thank the referee for his or her useful suggestions.

\section{References}

[1] R.L. Dobrushin, Teor. Veroyat. Primenenie. 18 (2) (1968) 201-229.

[2] R.L. Dobrushin, Funk. Anal. Pril. 2 (4) (1968) 44-57.

[3] D. Ruelle, Commun. Math. Phys. 9 (1968) 267-278.

[4] F.J. Dyson, Commun. Math. Phys. 12 (1969) 91-107.

[5] F. Spitzer, J. Func. Anal. 20 (1975) 240-255.

[6] W.G. Sullivan, Commun. Dublin Inst. Adv. Stud. Ser. A 23 (1975).

[7] S. Kalikow, Ann. Probab. 5 (1977) 467-469.

[8] J. Frohlich, T. Spencer, Commun. Math. Phys. 84 (1) (1982) 87-101.

[9] M.J. Miyamoto, Math. Kyoto Univ. 24 (4) (1984) 679-688.

[10] S.E. Burkov, Ya.G. Sinai, Uspekhi Math. Nauk 38 (1983) 205-225.

[11] M. Aizenman, J.T. Chayes, L. Chayes, C.M. Newman, J. Stat. Phys. 50 (1/2) (1988) 1-40.

[12] H. Berbee, Ann. Prob. 17 (4) (1989) 1416-1431.

[13] A.A. Kerimov, J. Stat. Phys. 72 (3/4) (1993) 571-620.

[14] A.A. Kerimov, Physica A 225 (1996) 271-276.

[15] A.A. Kerimov, J. Phys. A 31 (1998) 2815-2821.

[16] A.A. Kerimov, Physica A 258 (1998) 183-202. 